\title{
Methicillin Resistance and Biofilm Formation of Clinically Important Coagulase Negative Staphylococci
}

\author{
B. Subitha ${ }^{*}$ and J. Pavithra Devi ${ }^{2}$ \\ ${ }^{1}$ Department of Microbiology, Govt. Thoothukudi Medical College, \\ Thoothukudi - 628008, Tamil Nadu, India \\ ${ }^{2}$ Department of Microbiology, Govt. Stanley Medical College, \\ Chennai - 600001, Tamil Nadu, India \\ *Corresponding author
}

Keywords

CoNS, Methicillin resistance, Biofilm production

Article Info

Accepted:

20 May 2021

Available Online:

10 June 2021
Nowadays Coagulase-negative staphylococci (CoNS) are the most common causes of device related infections, especially in extreme age group of patients such as neonates and old age. The antibiotic resistance and biofilm production of CoNS produce negative impact on clinical outcome of patients, The study is therefore conducted to determine the antimicrobial susceptibility pattern and surface adherence property of CoNS isolated from various clinical specimens.. During the six months study period 46 CoNS were isolated from various clinical specimens Staphylococcus epidermidis (47\%) was the common isolate followed by Staphylococcus haemolyticus (24\%), and Staphylococcus saprophyticus (24\%). CoNS isolates showed maximum resistance to Ampicillin (76\%), Clindamycin(74\%), Cotrimoxazole (74\%) and Erythromycin(72\%). CoNS isolates revealed $100 \%$ sensitivity to Vancomycin. $72 \%$ of CoNS isolates showed Methicillin resistance and 61\% were biofilm producers by Congo Red Agar method. Methicillin resistance and Biofilm production of CoNS lead to increased morbidity and mortality in hospitalized patients. Aseptic management should be followed in intravascular devise insertion and other invasive procedures to reduce the spread of CoNS infection.

\section{Introduction}

Previously Coagulase-negative staphylococci (CoNS) are considered as non-pathogenic commensals of human skin and mucosa. With the advancement of medical sciences, especially with the increasing use of medical devices, the infections caused by CoNS are rapidly increased ${ }^{(1,2)}$. CoNS are an important causative agent for Neonatal septicemia worldwide $^{(2,3)}$. Commonly isolated species of CoNS include $S$. epidermidis $S$. haemolyticus, S. saprophytiicus, S. lugdunensis, S. warneri, S. cohinii, and S. hominis. ${ }^{(4)}$ Resistant to multiple antibiotics is commonly reported in CoNS. These strains are not only resistant to 
multiple antibiotics, but also act as reservoirs for drug resistance gene. Methicillin resistance (MR) among CONS is particularly important due to resistance to all beta-lactam agents and cross resistance to other anti-microbial classes. The resistance to methicillin and other betalactam antibiotics in both MR Staphylococcus aureus and MR-CoNS is primarily caused by the acquisition of the mec $\mathrm{A}$ gene, which encodes a modified penicillin-binding protein 2a that has a low binding affinity for all betalactam antibiotics. $(5,6,7)$

Biofilm production is considered as a main virulence determinant of CoNS and it protect them from host immune system and antimicrobial therapy. Biofilm consists of multilayered cell clusters embedded in a matrix of extracellular polysaccharide called polysaccharide intracellular adhesion (PIA).The development of the biofilm is begun with bacteria adhering to a biotic or an abiotic surface mediated by microbial surface components recognizing adhesive matrix molecules (MSCRAMMs) ${ }^{(6)}$. Then, the bacteria multiply to form a multilayered biofilm, associated with production of PIA which mediates cell to cell adhesion. The synthesis of PIA is mediated by the products of the intracellular adhesion (ica) operon. $(5,6,7,8,9)$. Detection of biofilm production and Methicillin resistance is very important for the treatment of infections that are caused by CoNS ${ }^{(10,11,12)}$. The present study is therefore conducted to determine the antimicrobial susceptibility patternand surface adherence property of CoNS isolated from various clinical specimens.

\section{Materials and Methods}

This prospective study was conducted at Thoothukudi Medical College over a period of six months. During the study period 46 coagulase negative Staphylococci were isolated from various clinical specimens.
The isolates were initially identified by colony morphology, Gram staining, catalase, slide and tube coagulase test acid formation from mannitol, Novobiocin sensitivity test and urease test. $^{(13,14)}$

Susceptibility to antibiotics were performed on Mueller-Hinton agar plates by KirbyBauer disc diffusion method. Zone diameter was measured and interpreted as per the Clinical and Laboratory Standards Institute (CLSI) guidelines.

\section{Minimum Inhibitory Concentration determination}

MIC of Cefoxitin \& Vancomycin was determined by an Epsilometer test (E test). A peptone water suspension of each CoNS isolate was adjusted to a McFarland standard of 0.5 and inoculated over the surface of the Mueller Hinton agar plate. The MIC strip was applied on the agar surface using sterile forceps. Then the plates were incubated for overnight at $37^{\circ} \mathrm{C}$ and the MIC was the point where the elliptical zone of growth inhibition intersected the MIC scale on the E test strip. The concentration range of antimicrobial on the $\mathrm{E}$ test strip corresponds to two fold dilutions in a conventional MIC method ${ }^{(15,16)}$.

\section{Detection of Methicillin resistance}

Methicillin resistance was detected by Cefoxitin Disc Diffusion Method and also by Cefoxitin E Test. ${ }^{(15,16)}$

Phenotypic characterization of biofilm formation by Congo Red Agar Method (17, 18, 19).

\section{Congo red agar method}

This test was performed on Congo red agar (CRA) plates as described by Freeman and coworkers and based on the property of Congo 
red to stain polysaccharides black. The composition of the medium are Brain Heart Infusion (37 gms/L), sucrose (50 gms/L), agar no.1 (10 gms/L) and congo red stain (0.8 gms/L). CoNS isolates were plated on congo red agar plate and were incubated at $37^{\circ} \mathrm{C}$ for $24 \mathrm{~h}$. The biofilm forming strains produced black colonies while non-forming strains developed red colonies.

\section{Results and Discussion}

During the study period 46 CoNS were isolated from various clinical specimens. Staphylococcus epidermidis $(47 \%)$ was the common isolate followed by Staphylococcus haemolyticus (24\%), Staphylococcus saprophyticus (24\%) and Staphylococcus lugdunensis (5\%).

CoNS isolates showed significant resistance to Ampicillin (76\%), Clindamycin (74\%), Cotrimoxazole (74\%) Erythromycin (72\%), Linezolid(6\%). CoNS isolates revealed 100\% sensitivity to Vancomycin. 33 (72\%) CoNS isolates showed Methicillin resistance.

Out of 46 CoNS isolates $28(61 \%)$ were biofilm producers by Congo Red Agar method

MIC of Vancomycin was $<2 \mu \mathrm{g}$. For MSCoNS MIC of Cefoxitin was $<2 \mu \mathrm{g}$ and for MRCoNS MIC of Cefoxitin was $>4 \mu \mathrm{g}$.

CoNS are considered one of the most common causes of the device related infections in the recent two decades, especially in extreme age group of patients such as neonates and old age. The increasing prevalence of CoNS infections is attributable to their increasing antibiotic resistance and their ability to form biofilms on foreign bodies such as intravascular catheters ${ }^{(17,18,19)}$. In the present study Staphlococcus epidermidis (47\%) was the common isolate followed by Staphlococcus haemolyticus (24\%) and Staphlococcus saprophyticus (24\%). This study correlates well with other studies conducted by Manjushree et al., Singh et al., Saroj Golia et al., and Vijayashri et al., who reported S.epidermidis was the most frequent isolate.

In the present study, antibiotic susceptibility testing showed all CoNS isolate were sensitive to Vancomycin and maximum resistance to ampicillin (76\%). Similarly Vijayashri et al., reported ampicillin resistance $(79 \%)$, and no resistance to Vancomycin. Manju shree et al., also reported all the CoNS isolates were susceptible to Vancomycin.

In the present study Methicillin resistance was observed in $72 \%$.This was concordant with Vijayashri et al., who reported Methicillin resistance in $72 \%$ of CONS and Surekha et al., reported $64.6 \%$ of Methicillin resistance. But lower Methicillin resistance 19.2 \%was pubhilished by Manju shree et al.,

The ability of biofilm formation seems to play an essential role in the virulence of coagulasenegative staphylococci (CoNS). ${ }^{(20)}$ Regarding the important role of genes associated with biofilm biomass production, the icaAD gene was found to be involved in biofilm formation, while the bap, fnbA, and cna genes were found to play a role in attachment to biotic or abiotic surfaces, which represents the first step of the process of biofilm formation. In the present study61\% were Biofilm producers by CRA method. 


\section{Chart.1}

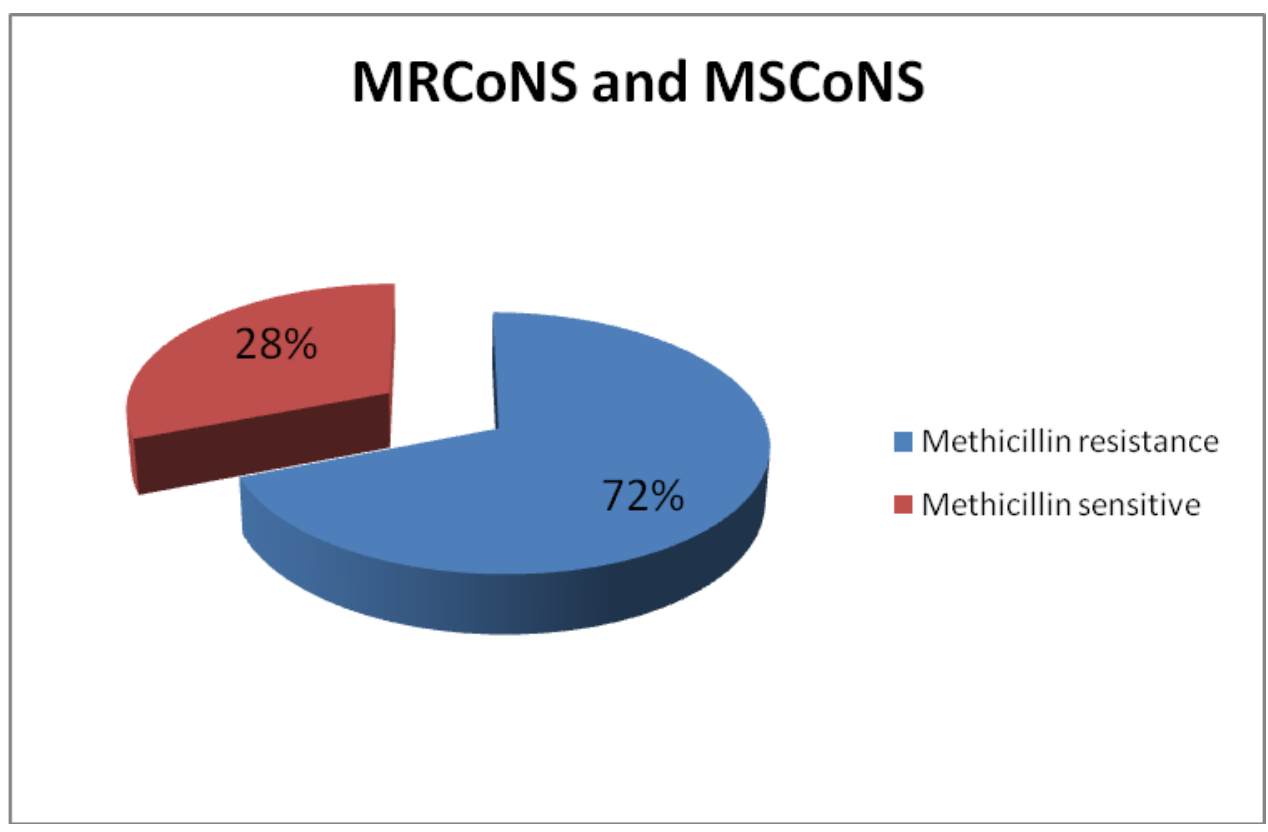

Fig.1 Congo Red Agar Method

Black colored colonies positive for biofilm production

Red colored colonies negative for biofilm production

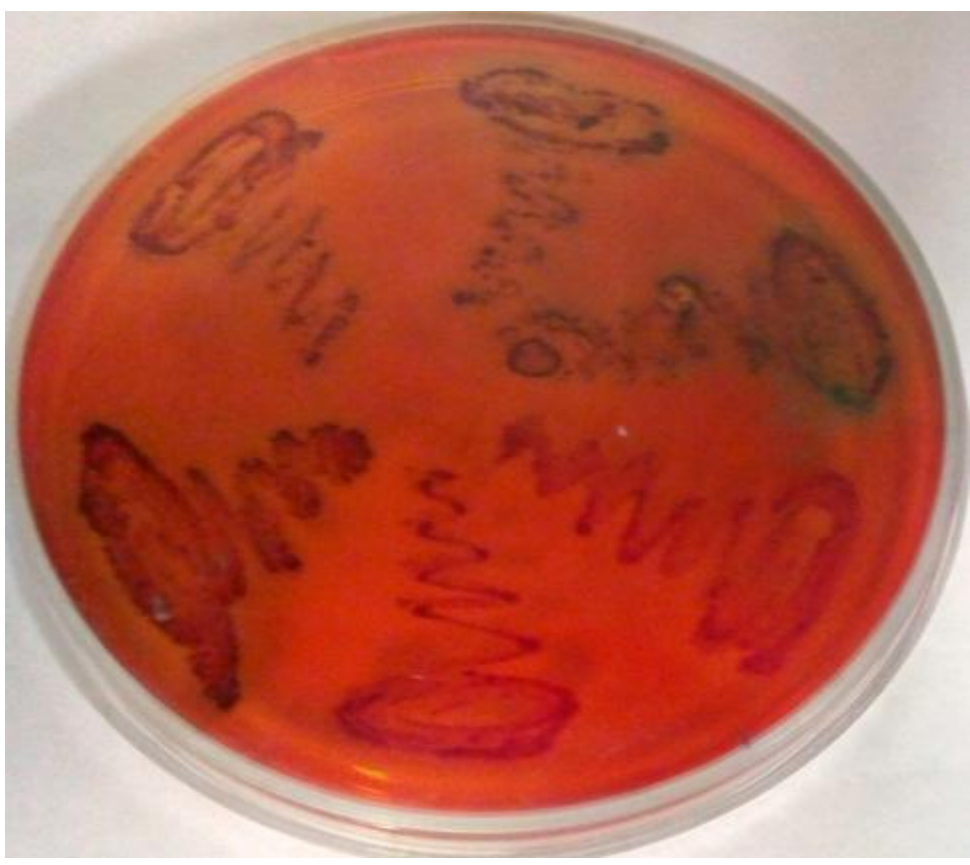


Fig.2 MIC determination for Vancomycin and Cefoxitin

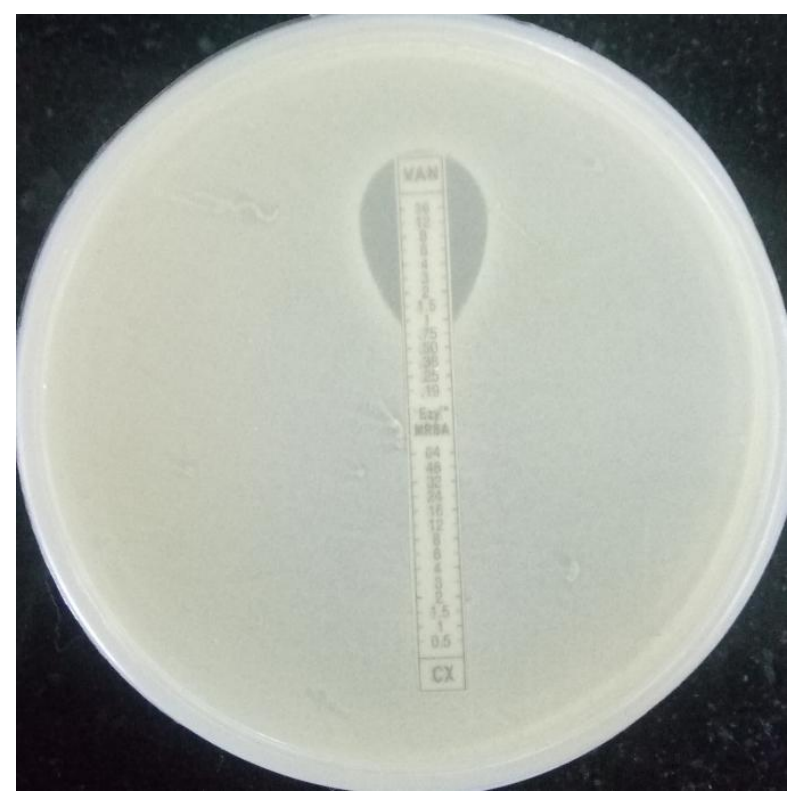

Similar findings were observed by Vijayashri et al., who reported (69\%) were Biofilm producers by CRA method. Mathur et al., and Riyaz Sheriff et al., identified that Tissue culture plate method was very sensitive and very specific method to detect the biofilm production.

Newer molecular methods available to detect genes responsible for biofilm formation. But in our study biofilm production was detected only by CRA method.

The high prevalence of biofilm producing strains and MR-CoNS demonstrated in this study indicates to follow the preventive measures to reduce the spread of MR-CoNS.

Increase in the use of medical devices, such as intravascular catheters and prosthetic devices, in neonates, will increase the prevalence of CoNS in infections. Biofilm-forming CoNS strains limit the immune system to counteract the infection. The Methicillin resistance and biofilm production of CoNS produce negative impact on clinical outcome of infected patients.

\section{References}

Bizzarro, Shabanova, Neonatal Sepsis 20042013: The Rise and Fall of CoagulaseNegative Staphylococci, The Journal of pediatric, Volume 166,Issue 5, 2015 pages 1193-1199

Elizabeth A. Marchant, Guilaine K. Boyce, Manish Sadarangani, and Pascal M. Lavoie, Neonatal Sepsis due to Coagulase-Negative Staphylococci Clinical and Developmental Immunology Volume 2013, 10 pages

Klingenberg C 1, Aarag E, Rønnestad A, Sollid J E, Abrahamsen,Coagulasenegative staphylococcal sepsis in neonates. Association between antibiotic resistance, biofilm formation and the host inflammatory response. Pediatr Infect Dis J. 2005 Sep;24(9):817-22.

Lok Bahadur Shrestha, Narayan Raj Bhattarai and Basudha Khanal, Antibiotic resistance and biofilm formation among coagulase-negative staphylococci isolated from clinical samples at a tertiary care hospital of 
eastern Nepal. Antimicrobial Resistance \& Infection Control. 20176:89.

Martini, Hörner, Investigation of biofilm formation in coagulase-negative staphylococci isolated from platelet concentrate bags, Rev Inst Med Trop Sao Paulo,2016,Volume 58

Mila V. S, Dr. S. Manjusree, Sri. Ashraf Perilacode, Speciation of the Coagulase Negative Staphylococci Isolated from Various Clinical Samples and their Antimicrobial Resistance Pattern at a Tertiary Care Hospital, JMSCR Vol||05||Issue ||03||Page 1906319068||March.

Muhammet Hamidullah Uyanik, Halil Yazgi, Kemalettin. Comparison of CoagulaseNegative Staphylococci Isolated from Blood Cultures as a True Bacteremia Agent and Contaminant in Terms of Slime Production and Methicillin Resistance.

Oliveira, De Lourdes Rs Cunha, Comparison of methods for the detection of biofilm production in coagulase-negative staphylococci, ResearchGate, 2010, Volume/page 260.

Rathanin Seng, Thawatchai Kitti, Rapee Thummeepak, Phattaraporn Kongthai, Biofilm formation of methicillinresistant coagulase negative staphylococci (MR-CoNS) isolated from community and hospital environments. PLOS ONE | https://doi.org/10.1371/journal.pone.01 84172 August 31, 2017

Riyaz Sheriff1, A Sheena2, Assessment of Biofilm Production in Clinically Significant Isolates of Staphylococcus epidermidis and Comparison of Qualitative and Quantitative Methods of Biofilm Production in a Tertiary Care Hospital, International Journal of Scientific Study |September 2016 | Vol
4 | Issue 6.

Roopa, Biradar, Incidence and Speciation of Coagulase Negative Staphylococcus Isolates from Clinically Relevant Specimens with their Antibiotic Susceptibility Patterns

Saroj Golia, Deepali Bhimacharya Telsang, Asha S. Kamath B, Speciation of clinically significant coagulase negative staphylococci and their antibiotic resistant patterns in a tertiary care hospital, International Journal of Research in Medical Sciences, 2015 May;3(5):1242-1246.

Shivanna, Sunkappa, The rising trend of coagulase-negative staphylococci in neonatal septicemia, Indian Journal of Pathology and Microbiology, Volume59/ issue 4 pages 510-514

Shreya Singh, Sujeesh Sebastian, Benu Dhawan, The Changing Face of Coagulase-Negative Staphylococci: Diagnostic And Therapeutic Challenges, Manipal Journal of Medical Sciences | June 2016 | Volume 1, 29-37.

Singh, Banerjee, Simple method for speciation of clinically significant coagulase negative Staphylococci and its antibiotic sensitivity/resistant pat-tern in NICU of tertiary care centre. www.biomedres.info

Surekha. Y. Asangi, Mariraj. J, Sathyanarayan. M. S, Speciation of clinically significant Coagulase Negative Staphylococci and their antibiotic resistant patterns in a tertiary care hospital. Int $\mathrm{J}$ Biol Med Res. 2011; 2(3): 735-739.

T Mathur, S Singhal, S Khan, D J Upadhyay, $T$ Fatma, A Rattan, Detection of biofilm formation among the clinical isolates of staphylococci: an evaluation of three different screening methods, Indian Journal of Medical Microbiology, (2006) 24 (1):25-9, 
Thawatchai Kitti, Rathanin Seng, Biofilm Formation of Methicillin-resistant Coagulase-Negative Staphylococci Isolated from Clinical Samples in Northern Thailand, J Glob Infect Dis. 2019 Jul-Sep; 11(3): 112-117.

Usha M G 1, Shwetha D C, Vishwanath G. Speciation of coagulase negative Staphylococcal isolates from clinically significant specimens and their antibiogram. Indian J Pathol
Microbiol. 2013 Jul-Sep;56(3):258-60. doi: 10.4103/0377-4929.120383.

Vijayasri Badampudi, S. S., Surya Kirani K R L, Rajyalakshmi, Coagulase Negative Staphylococcus; the Speciation and Biofilm Production of Coagulase Negative Staphylococcal Isolates from Clinically Significant Specimens and their Antibiogram. JKIMSU, Vol. 5, No. 2, April-June 2016.

\section{How to cite this article:}

Subitha, B. and Pavithra Devi, J. 2021. Methicillin Resistance and Biofilm Formation of Clinically Important Coagulase Negative Staphylococci. Int.J.Curr.Microbiol.App.Sci. 10(06): 606-612. doi: https://doi.org/10.20546/ijcmas.2021.1006.066 